$\begin{array}{llllllllllllllll}A & R & T & I & C & U & L & O & D & E\end{array}$

$\begin{array}{lllllllll}R & E & F & L & E & X & I & O & N\end{array}$

\title{
DiNÁMICAS DE LA DISCAPACIDAD PENSADAS DESDE EL LUGAR DE LAS FAMILIAS Y/O CUIDADORAS/ES: EXPERIENCIAS COMO EJE DE REFLEXIÓN PARA LA INCLUSIÓN.
}

DYNAMICS OF DISABILITY THINKING FROM THE PLACE OF FAMILIES AND

/ OR CAREGIVERS: EXPERIENCES AS A REFLECTION AXIS FOR INCLUSION

* Estudiante de octavo semestre de Trabajo Social. Miembro del sesocial. Miembro del se millero de investigación SODEFOR del Observatorio para el Desplazamiento Forzado. Universidad de Cartagena, Colombia.

* Estudiante de 8vo semestre de Trabajo Social. Miembro del semillero de investigación Cultura, Ciudadanía y Poder en Contextos Locales. Universidad de Cartagena, Colombia

\author{
Por: Angie Paola Menco Cadena* \\ Diana Paola Cantor Rueda**
}

Recibido: 15 de febrero de 2018 - Aprobado: 23 de abril de 2018

\begin{abstract}
RESUMEN
El presente documento pretende traer a discusión las dinámicas de las personas con discapacidad en su entorno familiar dirigido al papel de las cuidadoras y los cuidadores, instalando como referente la normativa que respalda y garantiza los derechos de dicha población. De acuerdo al planteamiento teórico, se realiza una aproximación a las realidades vividas por las personas con discapacidad, abordando casos específicos en lo comunitario y en lo organizacional, ello sugiere la necesidad de abordar aspectos cotidianos en los que se desenvuelven las personas para dar cuenta de cómo se vive la discapacidad. Además, se analizan las apuestas que se llevan a cabo en el Trabajo Social frente a la articulación de organismos, instituciones y sociedad para propiciar el fortalecimiento de las relaciones interpersonales de las personas con discapacidad, atendiendo a la socialización directa con su familia y su entorno. Por último, se evidencia que las experiencias en el marco de otra mirada de la discapacidad albergan la labor de las/os cuidadoras/es en el desarrollo personal y social de las personas con discapacidad; no obstante, este cuidado tiende a sobreponer la vida de la persona con discapacidad sobre las expectativas personales, sueños, deseos, necesidades, temores y aspiraciones de las/os cuidadoras/es.
\end{abstract}

Palabras clave: Derechos, Discapacidad, Experiencias, Familia.

\begin{abstract}
This document aims to bring to the discussion the dynamics of people with disabilities in their family environment addressed to the role of caregivers, installing as a reference the regulations that support and guarantee the rights of this population. According to the theoretical approach, an approximation is made to the realities lived by people with disabilities, addressing specific cases in the community and in the organizational, this suggests the need to address daily issues in which people unfold to account for how the disability is lived In addition, we analyze the bets that are carried out in the Social Work against the articulation of organizations, institutions and society to promote the strengthening of the interpersonal relationships of people with disabilities, attending to direct socialization with their family and their environment. Finally, it is evident that
\end{abstract}

Palubra No. 18. Agosto de 2018 
experiences within the framework of another view of disability are the work of the caregivers in the personal and social development of people with disabilities; However, this care tends to superimpose the life of the person with disability on the personal expectations, dreams, desires, needs, fears and aspirations of the caregivers.

Keywords: Rights, Disability, Experiences, Family.

La mirada dominante hacia el sujeto vulnerable, lo vulnera doblemente, lo vuelve dependiente, lo aísla, lo estigmatiza y hace de su diferencia acumulada una barrera para su inclusión.

NELIA TELLO

¿Especial? Lo que tengo de especial son unos padres y un entorno que han luchado porque sea lo más autónomo posible. PABLO PINEDA

\section{INTRODUCCIÓN}

T a sociedad es diversa en consideración de sus entramados sociales

y culturales, cada día se instalan nuevas maneras de ver y sentir la vida, en este contexto se evidencian las construcciones sociales de conocimiento que condicionan la configuración de las relaciones sociales, lo cual producirá inclusión o exclusión de grupos poblacionales, que en efecto implica el entendimiento o la incomprensión, la aceptación o el rechazo y el reconocimiento o invisibilización del otro/a.

En este panorama dicotómico, grupos minoritarios han trabajado y se han organizado por el reconocimiento y la reivindicación de sus derechos, las personas con discapacidad no han sido la excepción, las barreras existentes en la sociedad han suscitado la discusión de la inclusión de las personas con discapacidad; según el Observatorio Nacional de Discapacidad (2017) para el año 2005 de acuerdo al censo realizado por el DANE había 2.624.898 personas con discapacidad en Colombia, en lo que respecta a la posición que adopta el Estado, se ha reducido a una visión solidaria y proteccionista generando mayor exclusión en cuanto a derechos para esta población.

En ese sentido, hacen falta mecanismos y herramientas para la inclusión de una persona con discapacidad como un ciudadano/a con corresponsabilidad 
1 Frase tomada del libro: Aproximación a las realidades de las personas con discapacidad en Latinoamérica de Pilar Samaniego. y obligaciones, pero también como partícipe en el ejercicio de sus derechos. Entonces, se aboga por una noción que integre la normatividad, la familia, las interacciones sociales y las instituciones en el marco de la participación y representación, tal y como han defendido las personas con discapacidad: "Nada sobre nosotros sin nosotros".

Es importante en esa medida determinar que el desarrollo de este proceso de investigación está enfocado hacia la significación de las experiencias de las cuidadoras/es y personas con discapacidad en sus dinámicas cotidianas desde un enfoque interpretativo, acentuando los esfuerzos hacia la construcción de escenarios de inclusión y reconocimiento como sujetos de derechos. Desde allí se pretende mostrar los resultados de un trabajo teórico práctico, para tal fin resulta indispensable la entrevista como técnica de recolección de información partiendo de la recuperación de las experiencias con las/os cuidadoras/es a través de encuentros en escenarios locales como Flor del Campo y La María, desde lo organizacional con lideresas que representan los intereses de las personas con discapacidad, y desde lo comunitario a través de una red de personas con discapacidad que participan activamente en lo social.

Por tanto, inicialmente se abordará el significado de la discapacidad construido en la actualidad, no solo desde las instituciones especializadas, sino también reforzando cómo se construye por las personas que viven la discapacidad. Posteriormente, se enfatiza en la normatividad como respaldo y garantía de derechos para generar mejores condiciones de vida para la población con discapacidad. Luego se acentuará en las relaciones de las/os cuidadoras/es, la familia y las personas con discapacidad en lo concerniente a las dinámicas de su cotidianidad. Por último, se enfatiza la pertinencia de una propuesta desde el accionar del Trabajo Social en términos de acompañamiento profesional para promover espacios de reconocimiento de esta población y a sus familias en el abordaje de la discapacidad desde un enfoque holístico e integral.

\section{Otro mundo tan necesario como posible ${ }^{1}$ : La discapacidad construida en la significación de las realidades.}

Tr a palabra discapacidad presenta una mayor connotación de lo que su complejo término sugiere. Muchos conceptos han emergido, variadas instituciones han planteado un escenario en el que convergen distintos puntos desde los que se mira la discapacidad; sus denominaciones puestas en constante discusión han ido transformando el argumento, 
aseveraciones iniciales reducían la discapacidad a una problemática que implicaba directa y únicamente al individuo, mientras otras postulaciones reconocen la discapacidad como una relación entre los impedimentos físicos de la persona y las limitaciones impuestas por su entorno. En este punto, la Organización Mundial de la Salud hace una claridad al respecto desde la Clasificación Nacional del Funcionamiento de la Discapacidad y de la SaludCIF "Discapacidad es un término genérico que incluye: deficiencia o alteración en las funciones o estructuras corporales, limitación o dificultades en la capacidad de realizar actividades y restricción en la participación de actividades que son vitales para el desarrollo de la persona" (citado por el Departamento Nacional de Planeación, 2013, p.15).

Con objeto de lo planteado en la última adaptación de la definición de discapacidad enunciada por la CIF, es importante despejar la complejización al hablar del concepto común y personal que la gente tiene frente a la discapacidad muy alejada a la definición actual, al considerar "especiales" a las personas con discapacidad para reducirles a la inutilidad, reforzando así las pretensiones del pensamiento homogéneo que exigen a todos y todas cumplir con las características que les permita "encajar" correctamente en la sociedad. Esto conlleva a la determinación de un enfoque más amplio de la discapacidad que determina el compromiso de la institucionalidad en la medida de garantizar las mismas oportunidades, y desde la comunidad cuando la discapacidad se lleva a las instancias de una inclusión vista en la interacción con el otro/a.

La idea que se tiene de discapacidad debe trascender de esa concepción general, los sujetos que viven la discapacidad son seres como cualquier otro/a, con las mismas obligaciones, exigibilidad de derechos y de equidad. La esencia de una persona no se concentra en su corporalidad o en el funcionamiento de su organismo, sino en las capacidades y fortalezas construidas desde sus vivencias, pensamientos y emociones.

El proceso organizativo de las personas con discapacidad ha sido una labor ardua por la obtención del reconocimiento y la participación dentro de la sociedad en el marco de sus derechos, en cuanto a la salud, la educación, el empleo, la accesibilidad, la comunicación y el ejercicio político y de justicia. El conocimiento y dominio de la información respaldarán una vida digna como cualquier otro ciudadano y ciudadana, por tanto se hace necesario ahondar en el marco legal dejando todo un terreno amplio de discusión en materia de 
2 Aury Mulford. Abogada. Voluntaria de Corporación Mar Adentro. Entrevista, Cartagena de Indias, fecha: 7 de marzo de 2016

3 Raquel Molina. Promotora Social. Directora de la Corporación Mar Adentro. Entrevista, Cartagena de Indias, fecha: 14 de marzo de 2016

4 Shirly Howard. Ase-

sora en Fundación

Unidos Por un Futuro

Mejor. Shirly tiene 32

años y tiene focomelia

fecha: 30 de marzo de 2016 requerimiento y cumplimiento en todo lo referente al tema.

A la luz del enfoque legal: Problematizando las herramientas para su aplicabilidad

T a discapacidad compromete no solo al individuo que la experimenta, involucra a la familia y a todo su contexto, las instituciones y el Estado. Lo anterior, se refleja en el argumento de Aury Mulford ${ }^{2}$, quien presentó poliomielitis desde los primeros meses de nacimiento, ella hace referencia al escaso respeto de la condición humana, priorizando la discapacidad antes que a la persona junto con la falta de concientización más no de sensibilización respecto a la discapacidad.

Tras la reflexión expuesta cabe ahondar en la normatividad sobre discapacidad, la cual se rige y se establece cuando en Colombia por un constructo mundial tal y como resuelve la Secretaría de Integración Social de Bogotá (2012) por su impacto e implementación se adopta la Convención de las Naciones Unidas sobre los Derechos de las Personas con Discapacidad mediante la Ley 1346 en el año 2009, desde allí se presenta un enfoque de derechos y se comienzan a crear espacios de debate sobre el requerimiento de estos, consecuentemente se logra la Ley 1618 de 2013 que garantiza los derechos de personas con discapacidad a nivel nacional con el logro más relevante frente al dictamen de las responsabilidades directas hacia funcionarios, ministerios y entidades gubernamentales implicadas en la consecución de lo que declara la Ley (Fundación Saldarriaga Concha, 2013).

En contraposición, las aproximaciones de la regulación y cumplimiento de los derechos humanos de esta población son débiles, el Estado colombiano no ha logrado la consolidación de los mecanismos efectivos para su garantía. La problemática se instaura en la dificultad para demostrar una verdadera caracterización, el abordaje aún queda por discutir, el caso se agrava como argumenta la Corporación Mar Adentro ${ }^{3}$, perteneciente a la Red Cartagena Incluyente, cuando se habla de las caracterizaciones sin acoger a la población completa, aún hay personas que no tienen identificación o que no se encuentran sisbenizadas, esto resulta una barrera para su localización en los registros, lo que dificulta un estudio que contemple propuestas integrales de mejoramiento de la situación.

Como resultado de ello aún se percibe cierta insatisfacción en la población con discapacidad que exige mayor compromiso, no vendiendo sus derechos 
como una ayuda o servicio del Estado, así como lo señala Shirly Howard ${ }^{4}$ en mención de lo que compete a la normatividad, la existencia de las leyes no se sustentan en una inversión ya que no son generadoras de lucro. La garantía en este punto con lo mencionado por Shirly es la protección y restablecimiento de derechos.

En cuanto a la formulación y reformulación de leyes y artículos que protegen a la población con discapacidad la mayoría quedan solo en planteamientos. Se reconocen avances en materia de derechos; no obstante, aún se recurre a las tutelas o demandas para acceder a la salud, el fomento de escenarios para la organización y participación son insuficientes, la discriminación y las barreras arquitectónicas y actitudinales, además de las comunicacionales son frecuentes, esto acrecienta las brechas sociales y económicas existentes, lo cual devela un panorama de desafíos para la inclusión.

\section{Reflexiones en ejercicio de configuración desde las subjetividades de las familias: Trascendiendo en la comprensión de la discapacidad.}

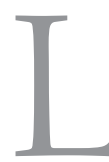

a familia catalogada como la institución social por excelencia ha sido un concepto difundido ampliamente en la sociedad, comprometida a ser perfecta desde la concepción, como afirma Aylwin y Solar (2002), de que todo comportamiento del individuo proviene a partir de la formación dada en ella, a causa de tal idealización se ha consumido la debida apariencia generadora de armonía y orden.

En cambio, desde la visión del construccionismo social Aylwin y Solar (2002) argumentan que la familia es un conjunto atravesado por concepciones y pensamientos diferentes que involucran las múltiples perspectivas que los individuos construyen en sus vidas de acuerdo a las experiencias que interpretan del contexto en que se encuentran inmersos. Se considera que lo que nutre a un individuo son las interacciones que crea con los demás, no solo en el entorno familiar, sino en otros medios de socialización; a partir de allí se entiende que la comunicación y el diálogo actúan como agentes potencializadores en la comprensión totalizadora de las distintas subjetividades y por ende de la familia en todo su alcance y profundidad.

De esta manera, si se logra un mayor acercamiento a los vínculos que se crean dentro de las dinámicas familiares se observan varias realidades, algunas personas con discapacidad, en ocasiones pueden representar para sus familias un obstáculo que impide el curso cotidiano de sus vidas como lo muestra

5 Linda Gómez. Ama de casa. Entrevista, Cartagena de Indias, fecha: 20 de marzo de 2016. 
6 Andrés Ávila. Estudiante, está en nove-

no grado y es atleta de la Liga de Parálisis Cerebral de Bolívar. Entrevista, Cartagena de Indias, Fecha: 17 de Marzo de 2016

7 Aury Mulford. Abogada. Voluntaria de Corporación Mar Adentro. Entrevista, Cartagena de Indias, fecha: 7 de marzo de 2016
Linda Gómez ${ }^{5}$, madre de Silfredo, de siete años de edad y con microcefalia, no va al colegio, manifiesta no querer estudiar porque se siente solo, además de llevar mucho tiempo sin asistir a terapias físicas; expresa Linda: "la silla de ruedas es muy pesada y él ha crecido y no puedo cargarlo hasta la fundación en la que le realizan las terapias".

Por el contrario, para otras puede verse como una forma particular de integración y una manera especial de asumir sus experiencias como sucede con Andrés Ávila ${ }^{6}$, tiene hipoacusia y secuelas de parálisis cerebral con espasticidad en los miembros superiores, su familia ha dedicado mucho esfuerzo y paciencia, han aprendido lengua de señas y han logrado comprender el mundo como lo ve Andrés.

Si bien la experiencia familiar apoya al desarrollo de la persona con discapacidad, sus procesos de socialización son fundamentales para su realización e integración en la sociedad, el aspecto de conocer y compartir con el otro/a hace que la fragmentación desaparezca y se produzca una verdadera inclusión, tal y como explica Aury Mulford ${ }^{7}$ referente a su etapa de aprendizaje escolar, ella estudió en una institución educativa oficial donde pudo contar con una infraestructura y accesibilidad, con apoyo y acogida de sus docentes y compañeros de clase por medio de espacios para su inclusión en un ambiente adecuado y de seguimiento a su proceso de desarrollo.

En concordancia con lo mencionado, cabría indicar que la discapacidad cambia la vida de quien la posee y por consiguiente la de sus familias, el estilo de vida, la forma de asumirla se convierte en una construcción que se consolida desde la familia en su relación con el medio que les influye, lo cual trae consigo la necesidad de adaptabilidad al cambio dentro del análisis y reconfiguración de los roles en el núcleo familiar, con la intención de suplir las demandas del cuidado. Reconociendo la relevancia del papel que desempeñan los familiares y/o cuidadoras y cuidadores en materia de discapacidad, en ellos recae un grado de responsabilidad para el desarrollo pleno de las personas que tienen a cargo, de hecho, la estabilidad emocional y económica que estos presenten recaerá directamente sobre las personas con discapacidad afectándoles de manera positiva o negativa.

Asimismo, la situación en la que se encuentre la familia contribuye también en la visión frente a la discapacidad, como menciona Samaniego (2006) que las desigualdades y los factores económicos y sociales son determinantes 
en la experiencia de vida de las personas con discapacidad, es por eso que la discapacidad es una experiencia particular, porque no todas las familias son iguales y porque los contextos en los que se encuentran inmersos son definitorios.

El medio social y económico son determinantes cuando se habla de discapacidad, puede que en ciertos casos el desarrollo de la persona sea más difícil debido a la falta de un salario estable, la apertura de información, de conocimiento y exigencia de sus derechos, y la falta de oportunidades, lo que en suma, hacen todo más complicado. Es manifiesto en las experiencias particulares a las que se pudo allegar en barrios vulnerables de la ciudad de Cartagena como La María y Flor del Campo, en estos lugares se rescataron experiencias particulares de personas con discapacidad y sus familias, la situación económica de las familias es inestable, a ello se agregan un sin número de problemáticas sociales que les afectan, además del debilitamiento de las relaciones dentro del vínculo familiar, la ausencia de los padres y madres dejando la responsabilidad del cuidado a los hermanos y hermanas. Los relatos son concretos, los factores sociales y económicos pueden incidir en el pleno desarrollo del individuo con discapacidad y su familia.

En este aspecto se destaca la interacción con las madres cuidadoras de personas con discapacidad vinculadas en torno a la liga deportiva de parálisis cerebral de Bolívar, una de ellas, presidenta de la organización, Nohora Orozco ${ }^{8}$, quién hace 21 años decide adentrarse en todo lo relacionado a la discapacidad a partir del nacimiento de su hija María José. En la relación que han construido se consolida el argumento basado en la comunicación y el diálogo, cobijando de esta manera la comprensión en lo familiar y la evidente influencia en la visión de María José como una joven que emprende decisiones para una vida independiente. Nohora ha complementado esto con un carácter fuerte en la vida de su hija, dándole siempre a conocer su igualdad en derechos y por supuesto en responsabilidades y deberes, sin desconocer que la base de todo este proceso ha sido la interacción con las demás personas, con y sin discapacidad.

Algo semejante ocurre con Emilse Ávila9 , la madre de Andrés, quien es atleta y ha sido apoyado por su madre para formar una vida que le ha garantizado el cubrimiento de sus necesidades básicas y aspectos de su personalidad como un ser sociable y activo en la sociedad. De hecho, Emilse siente que su relación con Andrés ha sido vital en su desarrollo, a pesar de no mediar palabras, las
8 Nohora, Directora de la Liga Deportiva de Parálisis Cerebral de Bolívar. María es secretaria de la liga con 21 años, tiene parálisis cerebral, practicó lanzamiento de bala y actualmente su deseo es ingresar a la Universidad. Entrevista, Cartagena de Indias, fecha: 25 de marzo de 2016

9 Emilse Ávila, ama de casa. Entrevista, Cartagena de Indias, fecha: 17 de marzo de 2016. 
expresiones corporales parecen darle mejor sentido a las situaciones por más simples que ellas parezcan. En el caso de Andrés, el acompañamiento de su madre ha sido una oportunidad para ella de cambiar en su rol, dice no tener ningún tipo de sinsabor al darse cuenta que dejó su vida por cuidar a su hijo, lo cual es algo que sin duda le hace estar orgullosa al ver los resultados en su cotidianidad.

Es pertinente aclarar que en general existen discapacidades diversas que en cierto sentido determinan la participación de la familia como intermediario fundamental o en otros casos no con la misma rigurosidad. Por consiguiente, el entorno familiar es facilitador de un espacio de autonomía y libertad, desafiando y rompiendo las barreras, el papel de la familia será promover el establecimiento de su forma de vida independiente; no obstante, se evidencian otras experiencias muy diferentes a la primera, en la cual las personas con discapacidad dependen del apoyo permanente de otras personas, quienes se entregan tiempo completo a su cuidado.

De acuerdo con lo argumentado y dando lugar a las concepciones personales que emergen en cuanto a la representación de las cuidadoras/es en la interacción de personas con discapacidad, con sus familias y el medio circundante, se hace hincapié en factores como el ánimo y la salud, como plantean Moreno, Rodríguez, Gutiérrez, Ramírez y Barrera, (2006) las personas en situación de discapacidad identifican en sus cuidadoras/es factores desfavorables tanto en su estado de salud como en su condición anímica, algunos se sienten extenuados, sobrecargados, insatisfechos y en los peores casos desesperados y fastidiados, muchos dedican tiempo completo al acompañamiento de las personas con discapacidad y se desvinculan de su vida personal y social, añadiendo que no cuentan con ingresos fijos para su sostenibilidad y la de sus cuidados.

Las/os cuidadoras/es reconocen que el rol que asumen cambió sus vidas de manera radical, de modo que anteponen las necesidades de su familiar hasta el punto de desatenderse y olvidar que ellas y ellos tienen una vida personal, descuidando también el aspecto emocional y espiritual. Por esta razón, se resalta la exigencia de una articulación más concreta y organizada entre la familia, la comunidad y las instituciones, vínculos que facilitarán la labor de la/el cuidador/a para encarar la discapacidad.

En el ámbito de las instituciones que prestan servicios de terapias y 
rehabilitación parece inevitable que no surjan cuestionamientos que demanden justificación de la labor que deberían llevar a cabo, un trabajo conjunto y asociado con la familia vinculado con papel del Estado. En este orden de ideas, el interrogante es conciso: ¿Es posible que las instituciones consideren incorporar a la familia en los procesos con sus miembros trascendiendo el modelo asistencial? La deficiencia en la relación entre instituciones y familias es notoria, la mayoría de instituciones cuentan con programas que incluyen a la familia pero solo en la rehabilitación y habilitación, tal y como refleja Nohora Orozco ${ }^{10}$ al relatar las realidades de las instituciones más reconocidas de Cartagena que prestan ese servicio "estos centros son máquinas de niños con discapacidad, no los enseñan a más, son proteccionistas, no les enseñan la independencia y la autonomía". Agrega Nohora que el derecho a la salud y la educación es vulnerado, la administración da licencia a IPS de garaje y las instituciones de educación no reciben personas con discapacidad porque en general no están preparados para hacerlo.

La intervención institucional debe estar enmarcada en el acercamiento a la discapacidad desde la familia y desde la persona con discapacidad, mejorando la calidad de la atención para el fortalecimiento de la labor de las/os cuidadoras/ es y enlazando los intereses de las familias, que genere mejores oportunidades para las personas con discapacidad (Buitrago, M., Ortiz, S. y Eslava, D., 2010), así como lo afirma Aury Mulford ${ }^{11}$ sobre el tiempo que se sintió culpable de haber nacido con discapacidad pensando en las dificultades que implicaba su cuidado y atención. Por lo que se demuestra un componente no solo de rehabilitación como normalmente se dirige, sino también de propiciar un ambiente que favorezca la inclusión en el empoderamiento de la familia y de la persona con discapacidad, sin recaer en el aprovechamiento de los beneficios que atañen a las cuidadoras/es o desde las mismas personas con discapacidad frente a su victimización y autocompasión.

Por esta razón, es menester rescatar el argumento de Núñez (2011) en cuanto a la importancia de poner objetivos dirigidos a la familia con una nueva visión orientada a la escucha y al intercambio de conocimientos por parte del profesional y de la familia, una relación dialéctica donde no prime solo la idea de cómo superar una limitación, en cambio, se intente conectar lo que siente y piensa la familia, viéndola al mismo tiempo a partir de las interpretaciones que se construyen para afrontar las necesidades que requiere la discapacidad.

Para finalizar, la familia debe proyectarse hacia la consolidación y promoción 10 Nohora Orozco,
Directora de la Liga
Deportiva de Parálisis
Cerebral de Bolívar.
Entrevista, Cartagena
de Indias, fecha: 25 de
marzo de 2016.
11 Aury Mulford.
Abogada. Voluntaria
de Corporación Mar
Adentro. Entrevista,
Cartagena de Indias,
fecha: 7 de marzo de
2016. 
de un ambiente que propicie un desarrollo social, digno y ético para la persona con discapacidad y sus integrantes, lo que desencadenará en un impacto positivo sobre la sociedad considerando espacios que estimulan y fortalecen a la familia desde su contacto con las instituciones sociales, para el empoderamiento en el proceso constante con la persona con discapacidad, descubriendo maneras de contrariar la exclusión, la discriminación, y la inoperancia del Estado.

\section{Apuesta desde el Trabajo Social en los procesos de inclusión de las personas con discapacidad y sus familias}

n la actualidad, el Trabajo Social se mueve en la comprensión de las realidades sociales vinculadas a problemas sociales y demandas que surgen de la construcción intersubjetiva de los actores sociales que lo experimentan, con el fin de modificar una situación problema en un espaciotiempo determinados. Sin embargo, aunque se reconoce la intención y pretensión de la disciplina por estar frente a las dinámicas sociales, se evidencia que el tema de la discapacidad desde su intervención ha sido tratado desde el enfoque médico y en ocasiones asistencialista.

En relación con lo previo, el rol del trabajador y la trabajadora social debe estar alejado del asistencialismo y de un sentimiento paternalista, de compasión y lástima que se considera distante del compromiso real que desde la disciplina debe promoverse, instalados en una perspectiva crítica del modelo social, que a su vez genere formas transversales de integrar los intereses de la población. En este contexto, se hace notoria la necesidad de una propuesta emergente que promueva el reconocimiento de las personas con discapacidad desde sus vivencias en las relaciones que establecen con el otro.

Esta propuesta asociada a un abordaje interdisciplinar como explicita Lorna (2014) es vital para el acompañamiento y respaldo en el Trabajo Social, el tema de la discapacidad precisa cultivar un mayor interés por la investigación y la intervención de los profesionales sociales en el medio cotidiano y natural de las experiencias de los individuos, sin aislarlos de su entorno y sus vínculos familiares y por supuesto con las instituciones y la estructura o sistema vigente.

El quehacer profesional como asegura Muyor (2011) inserto en los procesos que se llevan a cabo en la discapacidad, debe emprender proyectos incluyentes y significativos con la población, abriendo espacios de socialización y 
divulgación de propuestas que surjan de y para las personas con discapacidad, una mediación entre las personas, las instituciones y la ciudadanía. De modo que se traduzca en una visión holística de las distintas realidades sociales a partir del reconocimiento de las experiencias y de los significados que cada individuo le asigna a lo que vive. La propuesta de Muyor (2011) refiere el aprovechamiento de las posibilidades y realizaciones de los sujetos y sujetas en el que son vistos como agentes activos dentro del proceso de lucha por sus derechos.

En esa misma línea el Trabajo Social debe asumir la discapacidad en ámbitos como la investigación para la intervención social, la labor dentro de la institucionalidad, en asesoría y acompañamiento en una fundación u organización, en la participación de procesos organizativos, entre otros, logrando la identificación de las relaciones que entretejen las personas con discapacidad y su contexto, el cual demanda intereses específicos de un territorio.

Por tal motivo, Trabajo Social debe empezar a garantizar lugares de reconocimiento de las personas con discapacidad, esto desde el acompañamiento de la familia en la inserción y orientación de sus miembros en una comprensión integral de la discapacidad, fomentando así un espacio propicio para la construcción de una vida independiente para la persona con discapacidad.

En lo que refiere a la institucionalidad y a las instituciones que atienden a las familias y a las personas con discapacidad, el papel es determinante, Trabajo Social debe promover control social respecto a la institucionalidad, cuestionando el manejo de lo reglamentario y la aplicación en sus programas, políticas y leyes, asimismo en el desempeño de todos los funcionarios y de los responsables a cargo del tema de discapacidad en los procesos inclusivos de participación y buen vivir.

Acerca de las instituciones que ofrecen procesos de mejora de calidad de vida hacia las personas con discapacidad se puede expresar que hay asuntos por reforzar, las instituciones como el caso de las fundaciones contienen debilitamientos en el proceso psicosocial ante la persona con discapacidad y su familia. Los programas enfocados a las familias son muy someros, se nota la ausencia del fortalecimiento de la familia como una unidad que comprende un acontecimiento nuevo y diferente para ellos/as, pero que no determina solo el foco de atención hacia la persona con discapacidad, más bien que conecta la existencia de características actitudinales relevantes para 
mejorar el entorno familiar como el autoestima y el apoyo mutuo, todo esto con el fin de introducirse en la experiencia de la discapacidad.

En lo que respecta a las cuidadoras y cuidadores no se puede escatimar sus expectativas personales, al igual que su deseo de entregarse a su familiar. Teniendo en cuenta la importancia de la familia en el contexto de la discapacidad, Samaniego (2006) resalta: “(...) una situación de discapacidad no es netamente individual, es familiar y social, de ahí el énfasis en "personas con discapacidad y sus familias». Fortalecidos en lo individual y familiar avanzamos a lo comunitario, a lo social"(p.47), en este aspecto de doble línea se hace visible el acompañamiento del/la profesional social, quien debe gestar y promover escenarios de diálogo en los cuales la familia manifieste su apoyo al encargado/a del cuidado de la persona con discapacidad, en el fortalecimiento de las relaciones en el vínculo familiar y por consiguiente en la comunidad. El/ la profesional se debe insertar en las relaciones que la cuidadora y/o cuidador establecen con la persona con discapacidad para que se logre acrecentar los sentimientos de confianza, comprensión y compromiso ligado al tiempo que emplea en el cuidado, dejando a un lado sentimientos como vergüenza, inconformismo, cansancio y en peores casos fastidio.

A estas instancias es indispensable un punto fundamental, el fortalecimiento de los procesos organizativos de las personas con discapacidad y cuidadoras/ es, quienes se unen y se asocian para la consecución de objetivos, es en estos procesos organizativos donde se consolidan vínculos afines y puntos de mutuo acuerdo. Considerando lo anterior, se hace referencia a la urgencia de procurar un consenso en las personas con discapacidad, las cuidadoras/es, la sociedad en general, instituciones, fundaciones, corporaciones y el Estado mediante la reproducción de la cultura de la escucha y la comprensión, apoyada en reconocer las necesidades y demandas que imperan en toda la población con discapacidad, con la finalidad de impulsar procesos organizativos en representación de los intereses de estos.

En esta medida, el Trabajo Social debe fomentar la construcción de escenarios visibles para contribuir a un nivel más alto de conciencia en la sociedad, que asuma su corresponsabilidad en materia de respeto y posesión de unas luchas que no pertenecen solo a quienes tienen una determinada discapacidad, sino que entienda el valor de la unión de sus fuerzas.

En definitiva, las personas con discapacidad deben jugar un papel importante en el campo de conocimiento de la profesión, la concepción de la discapacidad 
debe estar enmarcada en la dignidad de la persona sustentado en el enfoque de derechos y el enfoque diferencial, esto es un paso para la participación y construcción de ciudadanía que esté al tanto de lo que sucede con el tema de discapacidad y también con lo que acontece en su entorno. Así pues, se ratifica la efectiva inclusión de las personas con discapacidad ya no pensándose solo desde sus luchas internas, sino en la participación de procesos sociales de base con un alcance en los ámbitos sociales, culturales, económicos y políticos. Resultado de esto, se señala la formación de ciudadanos que se identifiquen en un convivir tolerante y respetuoso, que se entiendan iguales en la diferencia, que se reconozcan pertenecientes a una población específica vinculante a lo social con todo un camino por construir y trascender.

\section{CONCLUSIONES}

Tr a discapacidad representa un desafío que involucra la participación y responsabilidad de toda la sociedad, la que debe propender hacia un eficaz L $ـ$ proyecto de inclusión comenzando con el cambio de los imaginarios, en la medida en que se discuta y reflexione cómo la población en general ha venido pensando la discapacidad.

Lo anterior, aboga por la configuración y compartición de una concepción distinta de la discapacidad a la luz de las experiencias de las/os cuidadoras/ es, quienes han asumido las vivencias de la discapacidad en la interacción con las dinámicas familiares y sociales, aproximándose a un proceso continuo de aprendizaje sobre lo que implica una vida con discapacidad. La investigación da cuenta de unos discursos y acciones que develan una crítica frente a la naturalización en cuanto a la dependencia, la revictimización, las barreras del entorno y la indiferencia de la población.

Las experiencias rescatadas de las familias de personas con discapacidad se tornan como insumo para repensar la discapacidad como una forma de vida común que obedece a la dignidad de ser humano antes de la situación de discapacidad, y que promueve una concepción que asuma que en la diversidad existen las personas con discapacidad, y que en su reconocimiento ellos/as también tienen derecho a ser incluidos y a participar en la construcción social de las realidades.

Así mismo las/os cuidadoras/es son el reflejo de una discusión actual sobre la dignidad y el derecho de las personas con discapacidad, ello implica superar el énfasis en el individuo y su enfermedad, con el fin de transcender a esferas 
como lo familiar, lo social, lo político, lo cultural y lo económico; solo a partir de la comprensión holística de estos aspectos será posible visibilizar el trasfondo de las luchas de las personas con discapacidad y sus familias como un asunto de carácter vinculante.

La labor que desempeñan las familias y en específico las/os cuidadoras/es en el acompañamiento de las personas con discapacidad requiere de una mayor atención, ya que su papel suele cargarse de la responsabilidad absoluta de la persona que tiene a su cargo, por lo anterior, se deben orientar los procesos en Trabajo Social a la reflexión sobre sus necesidades y demandas que trasciende, la actividad del cuidado, y se asientan en el desarrollo personal de la cuidadora o el cuidador.

En ese sentido, es inevitable aludir a la importancia de la familia como primer espacio de socialización para el desarrollo y desenvolvimiento en su medio de la persona con discapacidad; el aporte de las/os cuidadoras/es en la relación familiar y social brindan las posibilidades para que sean las personas con discapacidad quienes forjen y preparen espacios para la participación en la sociedad como actores visibles de sus derechos. La apuesta se fundamenta desde las voces de las familias y de las personas con discapacidad para lograr una articulación efectiva en la corresponsabilidad del Estado, las instituciones y/o fundaciones, y la comunidad, con el fin de promover un proyecto inclusivo de discapacidad.

En efecto, la sociedad, la familia, el colegio, los lugares de trabajo, los programas de gobierno y desde lo personal se debe trabajar por medios y estrategias para la inclusión apuntando mucho más a la conciencia, propiciando una actitud más crítica, que reconozca las voces de todos los actores sociales, ello implica que la sociedad al presentarse de cierto modo fragmentada por diferentes grupos y por ende diferentes motivaciones e intereses aparece al final como un conjunto, un mapa de complejidades y diversidad.

\section{REFERENCIAS BIBLIOGRÁFICAS}

ALWYN, Nidia y SOLAR, María. (2002). Trabajo social familiar. Chile. Ediciones Universidad Católica de Chile.

BUitrago, María Teresa, ORTIZ, Sandra, ESLAVA, Daniel. (2010). "Necesidades generales de los cuidadores de las personas en situación de discapacidad" En Investigación en Enfermería: Imagen y Desarrollo. Bogotá D.C. Editorial Pontifica Universidad Javeriana. Vol. 12(1), (enero-junio, pp. 
59-77).

DEPARTAMENTO NACIONAL DE PLANEACIÓN. (2013). CONPES SOCIAL 166. Política Pública Nacional de Discapacidad e Inclusión Social. Recuperado de: http://www.mincultura.gov.co/areas/ poblaciones/poblacion-condiscapacidad/Paginas/166.pdf. Fecha de consulta: 29 de diciembre de 2015.

FUNDACIÓN SALDARRIAGA CONCHA. (2013). Colombia, abre las puertas para garantizar los derechos de las personas con discapacidad con la puesta en marcha de la ley 1618 de 2013. Recuperado de: http://www.saldarriagaconcha.org/images/fsc/prensa/informes_especiales/2013/ discapacidad/0513_InformeLey\%201618.pdf. Fecha de consulta: 15 de diciembre de 2015.

LORNA, Sarah. (2014). El trabajo social en el área de discapacidad: Especificidades del rol profesional. Trabajo presentado en XXVII Congreso Nacional 11, 12 y 13 de septiembre de 2014. Recuperado de: https://issuu.com/faapss/docs/sidebottom_lorna. Fecha de consulta: 23 de enero de 2016. MORENO, María., RODRÍGUEZ, María., GUTIÉRREZ, Marybell., RAMÍREZ, Luz., y BARRERA, Olga. (2006). “¿Qué significa la discapacidad?” En Revista Aquichan. Colombia. Universidad de la Sabana. 6(1) (octubre, pp. 78-91).

MUYOR, Jesús. (2011). "La (con)ciencia del Trabajo Social en la discapacidad: Hacia un modelo de intervención social basado en derechos" En: Documentos de Trabajo Social. España. Colegio Profesional de Trabajo Social de Málaga. No. 49.(9-33 págs.).

Nú ÑEZ, Blanca. (2001). La relación familia-institución. Recuperado de: http://www. discapacidadyfamilia.com/pdf/Relacion_familia_y_profesionales.pdf Fecha de Consulta: 12 de Noviembre de 2015.

OBSERVATORIO NACIONAL DE DISCAPACIDAD. (31 de diciembre de 2017). Cómo van las estadísticas en discapacidad. Recuperado de: http://ondiscapacidad.minsalud.gov.co/Paginas/ Home.aspx. Fecha de consulta: 05 de Enero de 2018.

SECRETARÍA DE INTEGRACIÓN SOCIAL DE BOGOTÁ. (2012). Proyecto de Atención Integral a Personas con Discapacidad, Familias, Cuidadores y Cuidadoras-Cerrando Brechas. Recuperado de: http://old.integracionsocial.gov.co/anexos/documentos/2014_proy_boghum/proyecto_721.pdf Fecha de Consulta: 15 de Noviembre de 2015.

SAMANiEGO, Pilar. (2006). Aproximación a las realidades de las personas con discapacidad en Latinoamérica. Recuperado de: http://www.discapnet.es/Castellano/comunidad/websocial/ Recursos/Documentos/Tecnica/Documents/79216aa9238145598a639e0518e5d808Aproximac ionalarealidad.pdf Fecha de consulta: 19 de Noviembre de 2015. 